\title{
Cupins (Isoptera) Invasores da Rede Elétrica em Áreas Urbanas na Região do Alto Solimões, Amazônia Ocidental, Brasil
}

\author{
Agno Nonato Serrão Acioli ${ }^{\bowtie}$ \& Paulo Vitor Cassiano Oliveira
}

Universidade Federal do Amazonas, e-mail: acioli@ufam.edu.br (Autor para correspondência ${ }^{\bowtie}$ ), paulinho spo@hotmail.com.

\section{EntomoBrasilis 6(2): 150-156 (2013)}

Resumo. A ordem Isoptera (Insecta), popularmente conhecida como cupim é amplamente distribuída em toda a região Amazônica, com aproximadamente 238 espécies concentradas em quatro famílias. Este é o primeiro estudo de cupins na rede elétrica urbana de pequenas cidades no interior da Amazônia e teve como objetivo realizar um levantamento das espécies e associá-las ao dano causado nos postes urbanos. O estudo foi desenvolvido durante os anos de 2008, 2009 e 2011 em áreas urbanas das cidades de Benjamin Constant, São Paulo de Olivença e Atalaia do Norte, região do Alto Solimões, Amazonas. Foram vistoriados 1.614 postes, coletadas 129 amostras e identificadas nove espécies de cupins. A família Termitidae foi a mais abundante com $96,12 \%$, Rhinotermitidae com apenas 3,88\%. O gênero Nasutitermes foi o mais frequente, seus ninhos são construídos na parte superior dos postes. A espécie Nasutitermes acangussu Bandeira \& Fontes é a mais importante, causa prejuízos ao consumir a madeira dos postes e danos indiretos na fiação elétrica e acessórios de sustentação da fiação. Na parte inferior (base do poste) além da presença de galerias de Nasutitermes foi constatada a presença de galerias e ninhos de Coptotermes testaceus (Linnaeus), Heterotermes tenuis (Hagen) e Termes hispaniolae (Banks). Os postes de madeira devem ser substituídos por postes de concreto, as bases dos postes devem ser mantidas no limpo, a parte superior do poste e a fiação elétrica ao longo de toda sua extensão não devem ter contato com árvores ou objetos que favoreçam a infestação por cupins.

Palavras-Chave: Amazonas; Danos térmitas; Postes; Pragas urbanas.

\section{Termites (Isoptera) Invaders of Electrical Grid in Urban Areas of the Alto Solimões Region, Western Amazon, Brazil}

Abstract. The order Isoptera (Insecta), popularly known as termite is widely distributed throughout the Amazon region, with about 238 species concentrated in four families. This is the first study with termite in electrical grid in a small city in the interior of Amazon and aimed to perform a survey of the specie and relate them to the damage caused in the urban poles. The study was conducted during the years 2008,2009 and 2011 in urban areas Benjamin Constant, São Paulo de Olivença and Atalaia do Norte cities, Alto Solimões region, Amazon. Were inspected 1,614 poles, 129 samples were collected and identified nine species of termite. The family Termitidae was the most abundant with $96.12 \%$, Rhinotermitidae with only $3.88 \%$. The genus Nasutitermes was the most frequent their nests are built on top of poles. The Nasutitermes acangussu Bandeira \& Fontes was the most important because cause damage by eating the wood of the poles and causes indirect damages to electrical wiring and the support accessories. On the bottom (base of the poles) besides the presence of galleries of Nasutitermes was verified the presence of galleries and nests of Coptotermes testaceus (Linnaeus), Heterotermes tenuis (Hagen) e Termes hispaniolae (Banks). The wooden poles must be replaced by concrete poles, the bases of the poles must be kept clean, the top of the poles and electrical wirings along all the extension must not be in contact with trees or objects that favor termite infestation.

Keywords: Amazon; Posts; Termite damage; Urban pests.

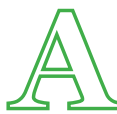
ordem Isoptera (Insecta), conhecida popularmente como cupim ou térmita (no Brasil), comejen (na Colômbia ePeru) e termites (em países de língua inglesa), é constituída por insetos sociais, com diferentes castas responsáveis por funções específicas dentro de uma colônia. Segundo Constantino (1998) são descritas para a Amazônia aproximadamente 238 espécies de cupins, ou seja, quase $9 \%$ do número de espécies descritas para o mundo e equivalente a aproximadamente $82 \%$ das espécies registradas para o Brasil. Para a Amazônia, assim como para o Brasil, são conhecidas quatro famílias de cupins: Termitidae, Kalotermitidae, Rhinotermidae e Serritermitidae.

Dentre os insetos sociais, RoBInson (1996) considera os cupins um dos mais bem sucedidos devido à longa vida de suas colônias e por utilizarem celulose como alimento, matéria abundante no sistema terrestre. Apesar disso, poucas espécies de cupins tem obtido sucesso na transição do ambiente natural para o ambiente urbano, e o sucesso de algumas espécies tem sido relacionado à sua habilidade em se adaptar às mais variadas condições existentes em meio urbano.

A composição das espécies e adaptabilidade dos Isoptera a novos ambientes varia entre regiões, altitudes e nichos. WALLER \& LA FAGE (1986) acreditam que a eliminação de competidores e predadores pelo homem facilita a proliferação dos cupins em áreas urbanas.

Das espécies adaptadasaoambienteurbanomuitas desenvolveram estratégias de voo que evitam a predação por pássaros e outros animais. Esses cupins realizam as revoadas ao entardecer, quando os seus predadores estão diminuindo suas atividades, e por serem pequenos e de coloração escura passam despercebidos em locais pouco iluminados (RoBINSON 1996).

Agência(s) de Finaciamento: Conselho Nacional de Desenvolvimento Científico e Tecnológico (CNPq), pelo apoio através do projeto de pesquisa MCT/CNPq/CT-Amazônia n ${ }^{0}$ 55/2008. 
Em áreas urbanas, os danos causados em construções são atribuídos a cupins pertencentes a três grupos: cupins de madeira seca, cupins subterrâneos e cupins de madeira úmida. No Brasil, a maioria dos estudos abordando a importância econômica dos cupins foi realizada na região Sudeste, sobretudo no Estado de São Paulo (Fontes \& Araujo 1999; Eleotério \& Berti-Filho 2000; Milano \& Fontes 2002a, 2002b). Para a Amazônia poucos são os estudos sobre cupins pragas, com destaque para os trabalhos desenvolvidos nas cidades de Belém - PA (BANDEIRA et al. 1989), Manaus - AM e Boa Vista - RR (BANDEIRA 1998). Conforme Constantino (2002) os gêneros Cryptotermes [Cryptotermes brevis (Walker)], Coptotermes [Coptotermes testaceus (L.)], Heterotermes, Cornitermes, Nasutitermes [Nasutitermes corniger (Motschulsky)] e Syntermes têm espécies com potencial de se tornarem pragas na região Amazônica.

Este trabalho teve como objetivo realizar um levantamento das espécies de cupins e descrever os danos causados na rede elétrica da área urbana dos municípios de Benjamin Constant, São Paulo de Olivença e Atalaia do Norte, na região do Alto Solimões, Amazonas, Brasil.

\section{MATERIAL E MÉTODOS}

Localização e Características Gerais da Área de Estudo. Este estudo foi realizado nos municípios de Benjamin Constant $\left(4^{\circ} 21^{\prime} \mathrm{S}\right.$ e $\left.70^{\circ} 2^{\prime} \mathrm{O}\right)$, São Paulo de Olivença $\left(3^{\circ} 27^{\prime} \mathrm{S}\right.$ e $\left.68^{\circ} 48^{\prime} \mathrm{O}\right)$ e Atalaia do Norte ( $4^{\circ} 20^{\prime} \mathrm{S}$ e $\left.70^{\circ} 9^{\prime} \mathrm{O}\right)$, as duas primeiras localizadas na calha do rio Solimões e a terceira na margem direita do rio Javari, todas na mesorregião do Alto Solimões, Estado do Amazonas, Brasil. O clima predominante na região Amazônica é do tipo equatorial (superúmido, Af segundo a classificação de Köppen), sem estação seca/semisseca, caracterizado por elevado índice pluviométrico, variando na mesorregião do Alto Solimões de $2.500 \mathrm{~mm}$ até $3.600 \mathrm{~mm}$, com média anual de $2.562 \mathrm{~mm}$; enquanto a temperatura média anual é de $25,7^{\circ} \mathrm{C}$. As classes de solos predominantes em Benjamin Constant são os Cambissolos (90\%) e os Gleissolos (10\%) e, muito provavelmente, em São Paulo de Olivença e Atalaia do Norte, uma vez que por similaridade de material de origem, do Alto Solimões, são de idades relativas bem mais jovens em relação àqueles profundos e bem drenados de grande parte das terras firmes da região Amazônica (Coelho et al. 2005).

Para os três municípios a energia elétrica é fornecida pela empresa Amazonas Distribuidora de Energia, do Sistema Eletrobrás. Os serviços de energia elétrica são basicamente oferecidos para a área urbana, que tem maior concentração de unidades consumidoras (residências) e densidade populacional (IBGE 2011). Porém, em municípios como Atalaia do Norte e São Paulo de Olivença mais da metade da população vive em comunidades na zona rural. Por outro lado, em Benjamin Constant mais de $60 \%$ da população vive na zona urbana, conferindo a esta cidade uma maior quantidade de serviços (Tabela 1).

Procedimentos de Coleta, Identificação e Análise dos Dados. Nos postes da zona urbana foi realizada uma procura por cupins no período entre agosto/2008 a abril/2011. Primeiramente todos os postes foram vistoriados e a coleta procedeu na faixa compreendida entre o nível do solo até 2,00 m de altura. Nos postes infestados, os cupins foram coletados manualmente, com auxílio de pinças, diretamente dos ninhos ou das galerias, externas ou internas. Procurou-se coletar indivíduos de todas as castas, que foram armazenados em frascos de $10 \mathrm{~mL}$ contendo álcool $80 \%$ e etiquetados. As características do local de coleta, presença ou não de vegetação no entorno do poste, material utilizado na construção dos ninhos e galerias, tipo de galeria (interna ou externa), presença ou não de ninho principal e o tipo de poste (concreto ou madeira) foram anotadas em caderneta de campo para auxiliar nas análises.

No laboratório, as impurezas das amostras foram removidas, gêneros e espécies foram identificados com auxílio de uma lupa e com a utilização de chaves (NICKLE \& Collins 1992; Constantino 1999, 2001) e descrições taxonômicas (Emerson 1925; MATHEws 1977; BANDEIRA \& FonTes 1979; Thorne et al. 1994). As espécies nãoidentificadas foram morfotipadas para posterior identificação. Todo o material foi depositado na Coleção de Insetos de Ciências Agrárias - CICA, do Instituto Natureza e Cultura/Universidade Federal do Amazonas - INC/UFAM, em Benjamin Constant Amazonas.

A análise estatística utilizada foi a distribuição de frequência das espécies, calculada com base na fórmula: $F=f X 100 / N$, onde: $F$ = frequência relativa $(\%) ; \mathrm{f}=$ frequência absoluta da espécie; $\mathrm{N}$ = número total de coletas efetuadas (BEIGUELMAN 2002).

\section{RESULTADOS E DISCUSSÃO}

Os postes da rede elétrica da região urbana das três cidades estudadas são de concreto e madeira. As espécies madeireiras utilizadas como postes na região são acariquara (Minquartia guianensis Aubl., Olacaceae), acapu (Vuouacapua americana Aubl., Caesalpiniaceae) e ipê roxo (Tabebuia sp., Bignoniaceae), todas conhecidas na região pela sua resistência aos fatores bióticos e abióticos.

Foram vistoriados 1.614 postes, dos quais $84,20 \%$ são de madeira e $15,80 \%$ são de concreto. Todos os postes vistoriados neste estudo estavam localizados nas áreas urbanas das três cidades e representam 48,02\% do número total de postes distribuídos nas áreas urbana e rural dos três municípios. Nos postes vistoriados foram coletadas 129 amostras e identificadas nove espécies de cupins, pertencentes às famílias Rhinotermitidae e Termitidae, as mais frequentes em estudos sobre cupins na Amazônia (Ackerman et al. 2009; Acioli \& Oliveira 2010).

Na Figura 1, observa-se que o maior número de postes vistoriados foi em Benjamin Constant (806), seguido de São Paulo de Olivença (530) e Atalaia do Norte (278). Em todos os postes infestados foram capturados cupins, sendo que o maior número de amostras coletadas foi em Benjamin Constant (50), seguido de Atalaia do Norte (44) e São Paulo de Olivença (35). Postes com o sinal de infestações, mas sem a presença de cupins, também foram computados e, neste caso, para Benjamin Constant foram registrados 59 postes, seguido de São Paulo de Olivença e Atalaia do Norte com 49 e 27 postes, respectivamente.

Estes resultados revelam que apenas $16,36 \%$ dos postes estavam danificados por cupins, mas a coleta só foi possível em 7,99\% dos postes. Mesmo com a baixa infestação de cupins nos postes, os

Tabela 1. Informações e características dos serviços prestados pela Amazonas Distribuidora de Energia para os três municípios estudados.

\begin{tabular}{|c|c|c|c|c|}
\hline \multirow[b]{2}{*}{ Cidades } & \multicolumn{4}{|c|}{ Informações/Características } \\
\hline & Extensão da rede (km) & $\begin{array}{c}\text { Unidades } \\
\text { consumidoras (casas) } \\
\end{array}$ & Postes & $\begin{array}{l}\text { Habitantes/ } \\
\text { Município }^{*}\end{array}$ \\
\hline Atalaia do Norte & 23,00 & 1.198 & 593 & 15.149 \\
\hline Benjamin Constant & 89,90 & 4.890 & 1.876 & $33 \cdot 391$ \\
\hline São Paulo de Olivença & 57,00 & 2.950 & 892 & 31.426 \\
\hline Total & 189,90 & 9.038 & $3 \cdot 361$ & 79.966 \\
\hline
\end{tabular}

* Dados obtidos do IBGE, 2011. 
prejuízos são relevantes em função de sua intensidade. Somente em Atalaia do Norte é que o número de postes com sinais de infestação foi inferior ao número de amostras coletadas com cupins. Mas, esta é a cidade que tem a menor área urbanizada dentre as três e, portanto, maior proximidade com áreas florestais, capoeiras, sistemas agroflorestais e campos.

Outros fatores que podem contribuir para a colonização e o estabelecimento de algumas espécies de cupins é a presença de pequenas áreas verdes no interior da região urbana, casas com quintais grandes e arborizados e a madeira utilizada na construção das residências são muito comuns na região. Pois, segundo Costa-Leonardo (2002), a adaptação de algumas espécies de cupins ao meio urbano depende da densidade populacional nas áreas naturais vizinhas à área urbana, tipo de solo e vegetação, além do material utilizado nas edificações.

Na Tabela 2, verifica-se que o maior número de espécies foi encontrado no município de Atalaia do Norte (8), seguido de Benjamin Constant (7) e São Paulo de Olivença (5). A família Rhinotermitidae está representada pelas espécies $C$. testaceus e Heterotermes tenuis (Hagen). Em Benjamin Constant foram registradas as duas espécies, enquanto que em São Paulo de Olivença foi registrado somente $C$. testaceus e nenhuma das duas em Atalaia do Norte. Mesmo com a baixa frequência das duas espécies constatada neste estudo, é de conhecimento que sua ocorrência é ampla e ocorre em toda a região Amazônica (Constantino 2002). Estas duas espécies tem sido registradas causando danos em plantios florestais e agrícolas e, também, em áreas urbanas em várias regiões do Brasil (BANDEIRA 1981; Nogueira \& Souza 1987; Abreu \& Jesus 2004; Costa-Leonardo 2002).

Os ninhos de $C$. testaceus e $H$. tenuis foram encontrados na base dos postes envolvidos por vegetação, normalmente se estendiam até aproximadamente 1,00 m de altura do solo. As galerias de $C$. testaceus estavam localizadas em frestas preenchidas com argila úmida; enquanto a espécie $H$. tenuis constrói galerias internas, em frestas ou sob uma fina camada de madeira, e externas, normalmente construídas de barro, localizadas próximas ao solo.

Para a família Termitidae foram encontradas sete espécies, isto significa $77,78 \%$ do total do número de espécies registrado neste

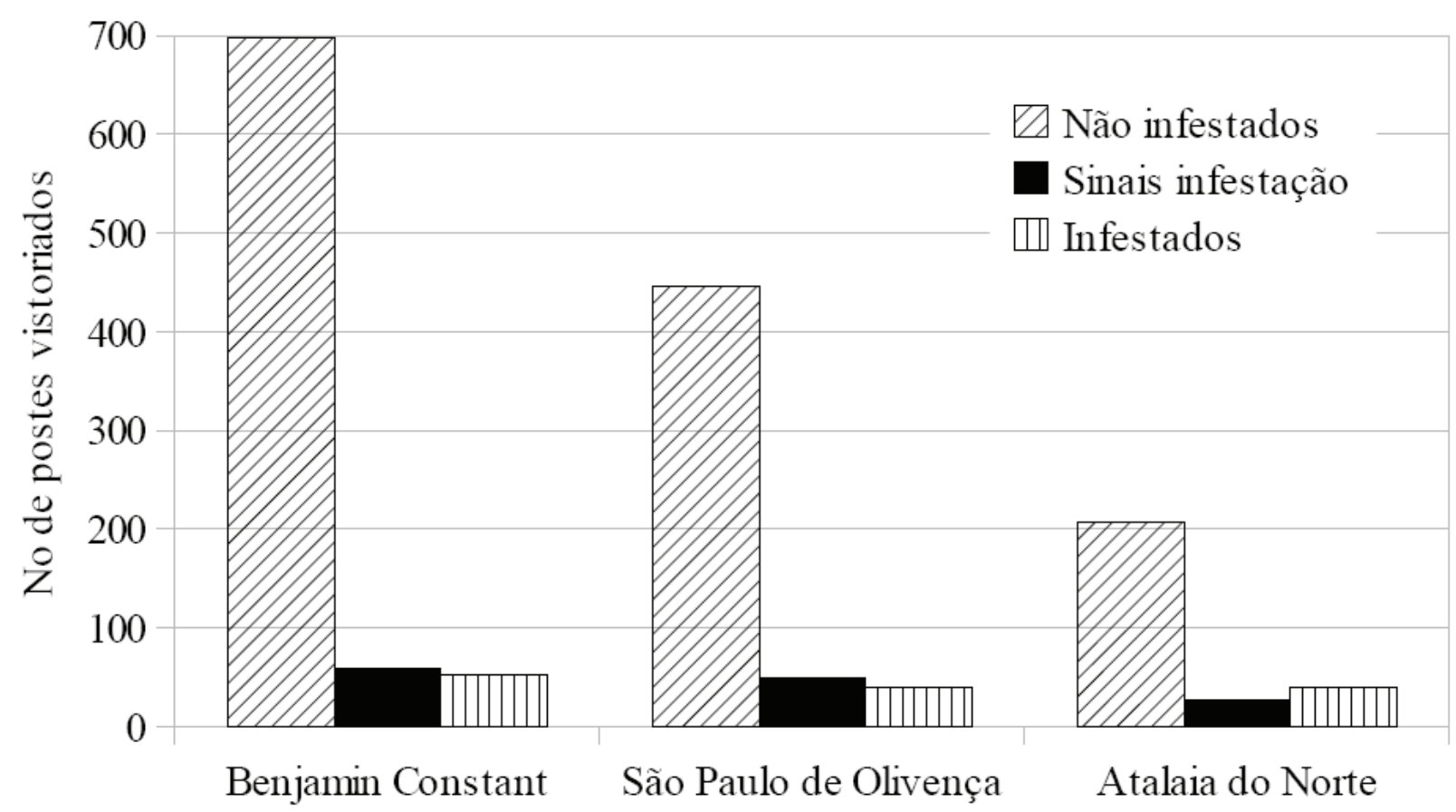

Figura 1. Número de postes vistoriados nas cidades de Atalaia do Norte, Benjamin Constant e São Paulo de Olivença.

Tabela 2. Quantidade de amostras (N) e frequência (F (\%)) das espécies/morfoespécies de cupins encontradas nos postes da rede elétrica das cidades de Benjamin Constant, São Paulo de Olivença e Atalaia do Norte na mesorregião do Alto Solimões, Amazonas Brasil.

\begin{tabular}{|c|c|c|c|c|c|c|c|c|}
\hline \multirow{2}{*}{$\begin{array}{c}\text { Família/Gênero/ } \\
\text { Espécie }\end{array}$} & \multicolumn{2}{|c|}{ Benjamin Constant } & \multicolumn{2}{|c|}{ São Paulo de Olivença } & \multicolumn{2}{|c|}{ Atalaia do Norte } & \multicolumn{2}{|c|}{ Total } \\
\hline & $\mathbf{N}$ & F (\%) & $\mathbf{N}$ & F (\%) & $\mathbf{N}$ & F (\%) & $\mathbf{N}$ & F (\%) \\
\hline Rhinotermitidae & 3 & 6,00 & - & - & 2 & 4,55 & 5 & 3,88 \\
\hline Coptotermes testaceus & 2 & 4,00 & - & - & 2 & 4,55 & 4 & 3,10 \\
\hline Heterotermes tenuis & 1 & 2,00 & - & - & - & - & 1 & 0,78 \\
\hline Termitidae & 47 & 94,00 & 35 & 100 & 42 & 95,45 & 124 & 96,12 \\
\hline Nasutitermes acangussu & 21 & 42,00 & 10 & 28,57 & 6 & 13,63 & 37 & 28,68 \\
\hline N. ephratae & 9 & 18,00 & 7 & 20,00 & 1 & 2,27 & 18 & 13,95 \\
\hline$N$. nigriceps & 12 & 24,00 & 11 & 31,43 & 18 & 40,91 & 41 & 31,78 \\
\hline N. surinamensis & - & - & - & - & 1 & 2,27 & 1 & 0,78 \\
\hline Nasutitermes sp. A & 1 & - & 1 & 2,86 & 9 & 20,45 & 11 & 8,58 \\
\hline Nasutitermes sp. C & - & - & - & - & 5 & 11,36 & 5 & 3,88 \\
\hline Termes hispaniolae & 4 & 8,00 & 6 & 17,14 & 2 & 4,56 & 12 & 9,30 \\
\hline Total & 50 & 100 & 35 & 100 & 44 & 100 & 129 & 100 \\
\hline
\end{tabular}


Trabalho. Destas, seis pertencem à subfamília Nasutitermitinae e apenas uma à subfamília Termitinae. Entre as espécies pragas da família Termitidae, Constantino (2002) relaciona 47 espécies, das quais 32 pertencem à subfamília Nasutitermitinae e 14 ao gênero Nasutitermes. Para a Amazônia, o gênero Nasutitermes é a principal praga em estruturas de edificações urbanas (BANDEIRA et al. 1989).

A identificação da espécie $N$. nigriceps ainda é motivo de dúvida. Mathews (1977) alerta que os espécimes identificados como $N$. nigriceps são muito próximas em semelhança aos espécimes N. acajutlae e N. macrocephalus. Constantino (2002) reforça que os espécimes identificados como $N$. nigriceps na América do Sul provavelmente sejam N. acajutlae ou N. macrocephalus. Mas, neste estudo, são consideradas a proposta de THorne et al. (1994) que separa distintamente $N$. acajutlae de $N$. nigriceps e as descrições e figuras em MathEws (1977).

As espécies Nasutitermes acangussu Bandeira \& Fontes, Nasutitermes ephratae (Holmgren) e Nasutitermes nigriceps (Haldeman) são as mais frequentes e, juntas, perfazem $74,41 \%$ do total de amostras coletadas.

A espécie $N$. nigriceps tem a maior frequência (31,78\%) e predomina em Atalaia do Norte com 40,91\% das amostras coletadas. Esta espécie tem sido relacionada como praga agrícola na Amazônia brasileira (Mill 1992 apud BANDEIRA 1998) e pela primeira vez está sendo relacionada como espécie praga em região urbana. A área urbana de Atalaia do Norte é muito pequena, arborizada e com corredores verdes em contato com a vegetação florestal, isto pode facilitar a permanência ou a colonização da região urbana por esta espécie. Enquanto a espécie N. acangussu, tem a segunda maior frequência $(28,68 \%)$ encontrada neste estudo, é bastante comum na região Amazônica. Conforme BANDEIRA (1998) é uma das principais pragas de postes da rede elétrica em Manaus - AM. Nas duas cidades mais urbanizadas, Benjamin Constant e São Paulo de Olivença, $N$. acangussu é a principal espécie encontrada nos postes da rede elétrica, confirmando desta forma sua importância como cupim praga no interior da região Amazônica. A espécie N. ephratae, a terceira mais frequente $(13,95 \%)$, é a terceira em predominância em Benjamin Constant e São Paulo de Olivença, mas tem baixa frequência em Atalaia do Norte, espaço ocupado pela espécie Nasutitermes sp. A. Vale destacar que N. ephratae e N. corniger são muito semelhantes morfologicamente, mas a distinção entre as duas espécies é possível com base na arquitetura externa dos ninhos (THORne 1980) e nos caracteres morfológicos (Nickle \& Collins 1992). Já a subfamília Termitinae está representada pela espécie Termes hispaniolae Banks presente nos postes das redes elétricas das três cidades, mas com maior frequência em São Paulo de Olivença.

Os postes de madeira, que totalizam quase $85,00 \%$ da rede elétrica, são mais suscetíveis ao ataque de cupins do que os postes de concreto. Durante as coletas observou-se que muitos estavam situados entre árvores, com a base no interior de vegetação e o contato da fiação elétrica com galhos de árvores (Figura 2). Esse quadro de situações facilita a infestação por cupins em postes nas zonas urbanas.

Os ninhos do gênero Nasutitermes foram encontrados predominantemente na parte superior dos postes. Nesta região o local mais comum é justamente nas peças que fixam os cabos elétricos aos postes (Figuras 3 e 4). Os ninhos normalmente são grandes, envolvem cabos, reatores, aparelhos fotoelétricos, roldanas, hastes de armação das roldanas, braços da luminária, cintas de aço e as cruzetas de madeira, usadas em postes tanto de madeira como de concreto. Esses componentes e estruturas envoltas pelo ninho estão sujeitos ao processo de decomposição, seja pela atuação dos cupins (em caso de peças de madeira ou mesmo de plástico) ou pela oxidação através de ácidos orgânicos (no caso de peças metálicas). Tanto em postes de madeira como de concreto o ninho está conectado ao solo por galerias, cujo objetivo é proteger os indivíduos responsáveis pela coleta de alimento, neste caso, nas proximidades da base dos postes. Também foram observadas galerias construídas nas fiações elétricas com objetivos de alcançar árvores que estavam em contato com os fios, muito provavelmente em busca de alimento.
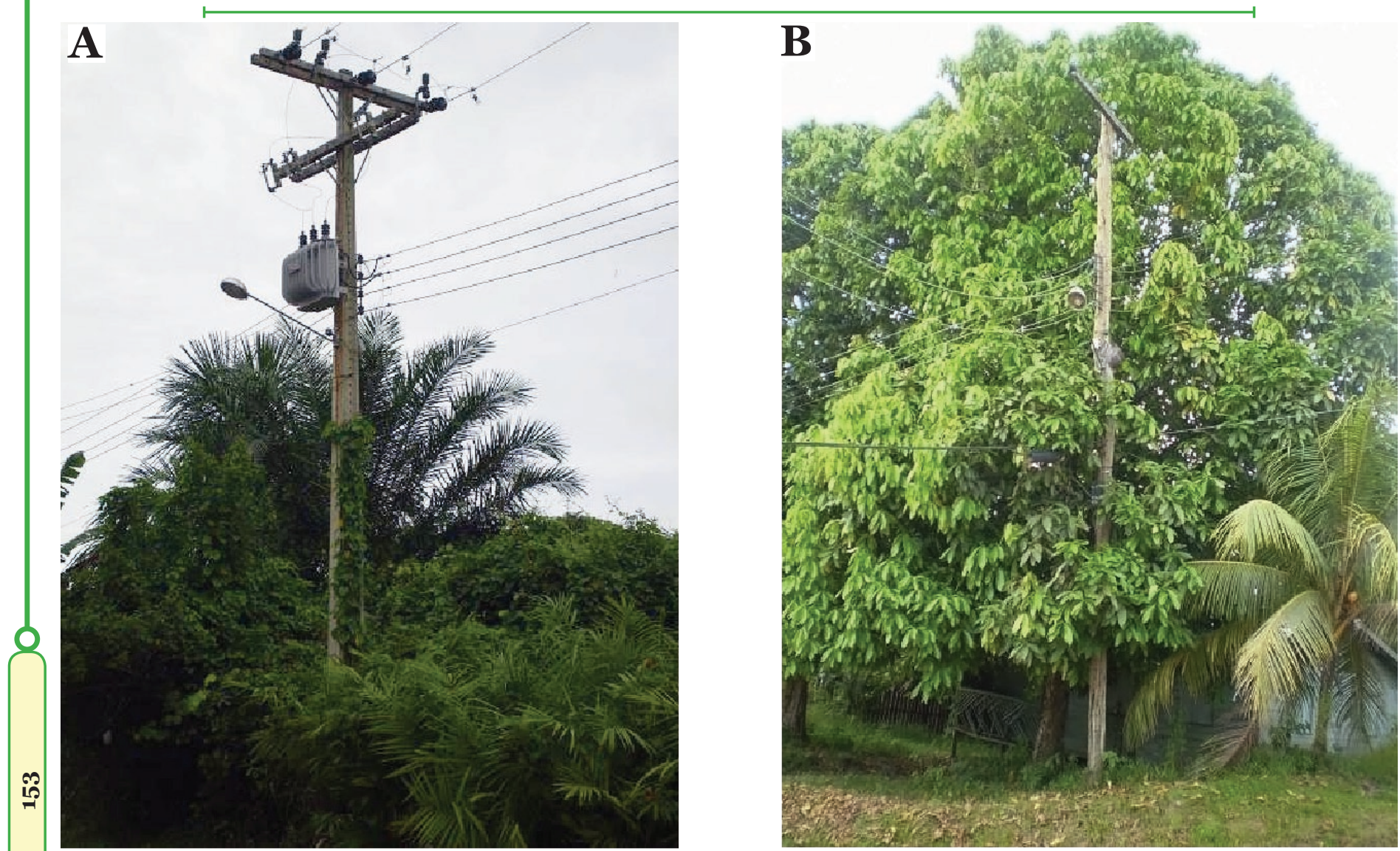

Figura 2. Aspecto geral mostrando a vegetação no entorno da base de postes (A) e o contato da arborização urbana com postes da rede elétrica (B) em Benjamin Constant, AM. 

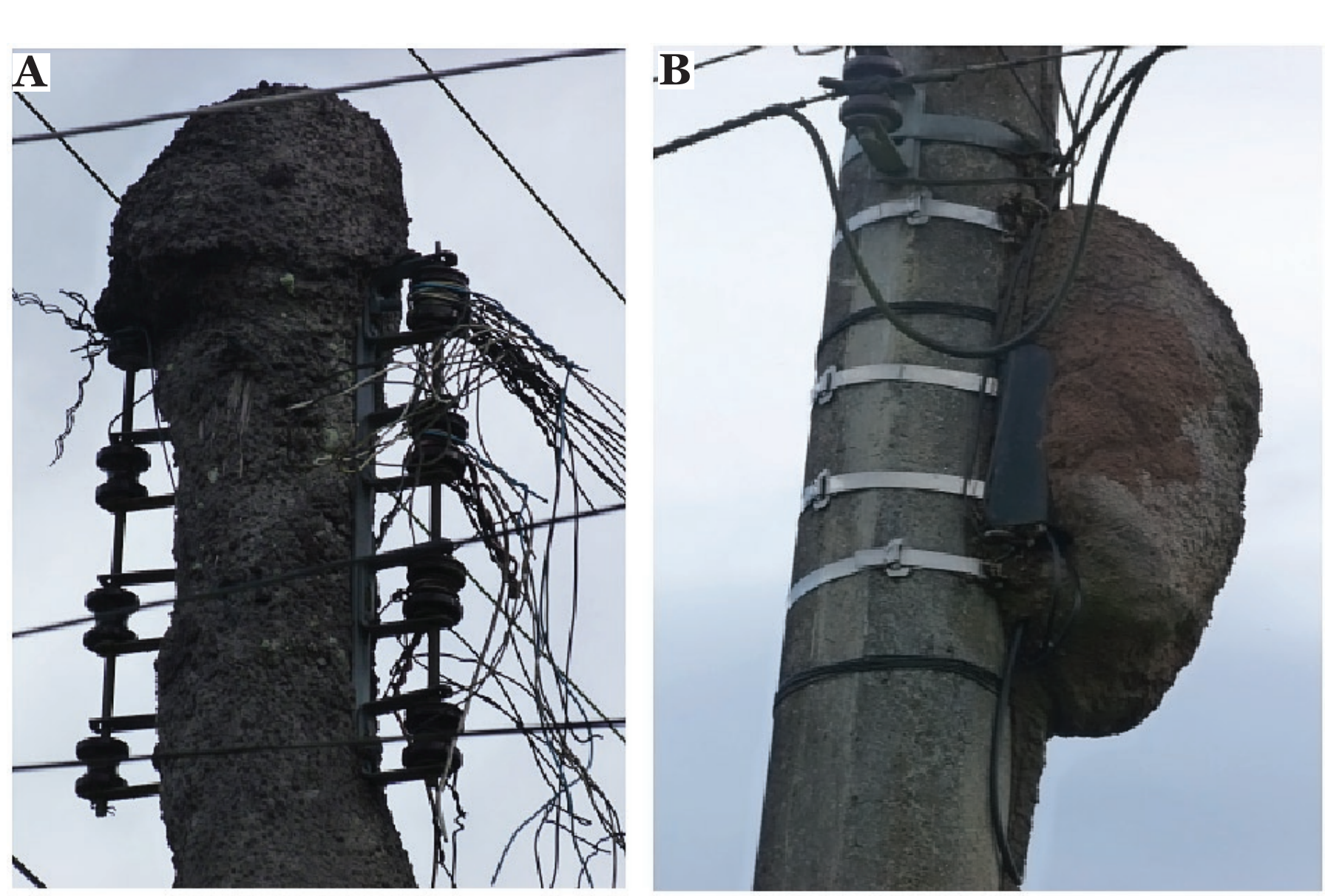

Figura 3. Ninho de N. acangussu (A) localizado no topo de um poste de madeira e ninho de N. ephratae (B) localizado em poste de concreto a aproximadamente 3,om de altura.
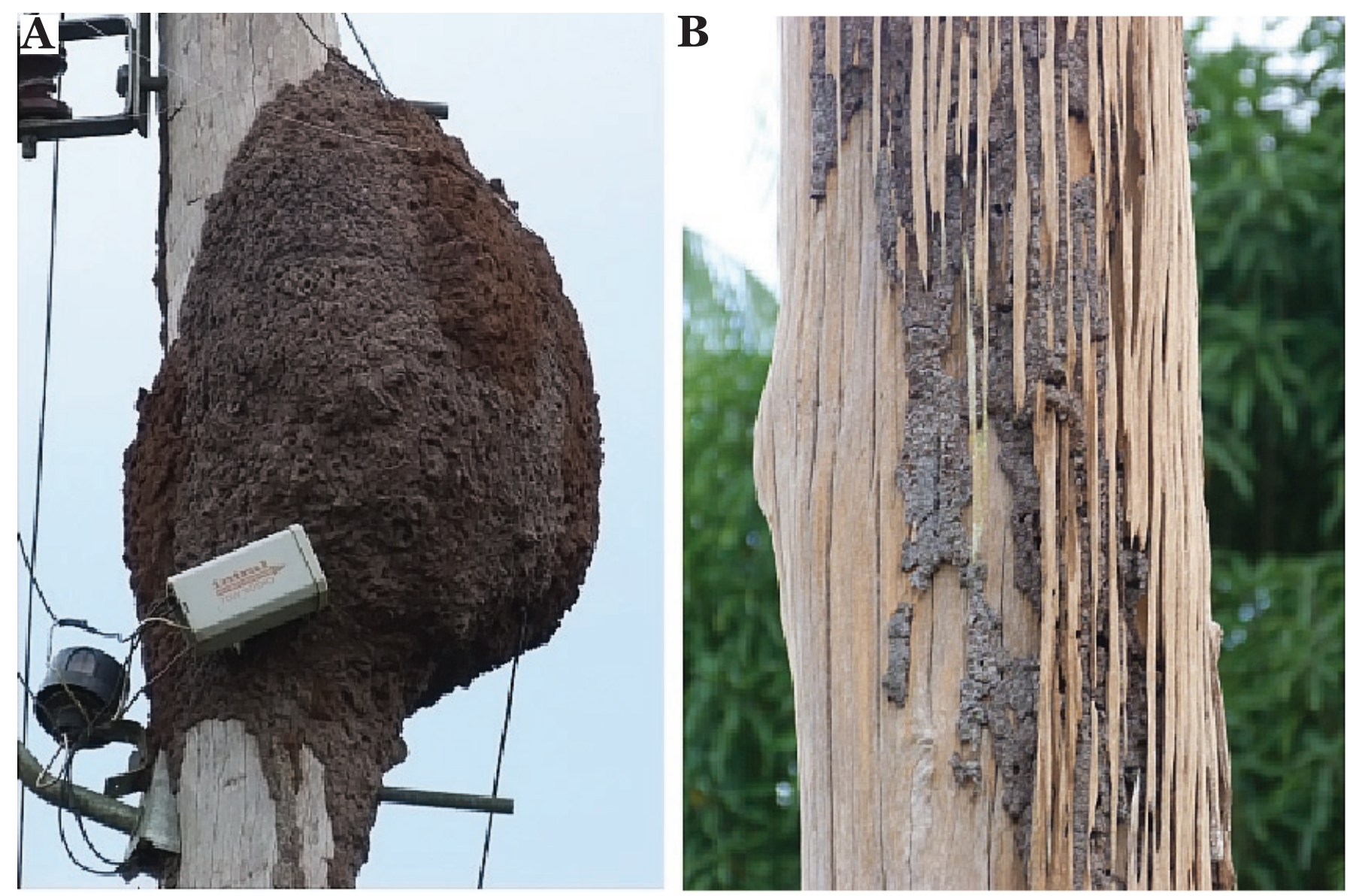

Figura 4. Postes de madeira da rede elétrica atacados por N. nigriceps (A), localizado próximo ao topo do um poste, e danos causados por N. acangussu (B). 
Nos postes de madeira o ataque normalmente é muito severo (Figura 4B) e o estrago causado pelo consumo torna-os propícios a tombamentos, situação muito temida por alguns moradores, conforme depoimentos relatados durante as atividades de coletas deste estudo.

Para T. hispaniolae, não foram observados ninhos conspícuos nos postes, mas galerias constituídas de um material mais escuro e bem visível envolvendo a região basal dos postes. As colônias não eram grandes, mas contribuíam direta ou indiretamente para a decomposição dos postes, através de seu consumo ou proporcionando ambientes favoráveis para a infestação de outras espécies de artrópodes ou fungos que atuam também no processo de decomposição dos postes, diminuindo assim sua vida útil. Segundo Constantino (1999), o gênero Termes ocorre em vários tipos de habitat, alimentam-se de madeira semidecomposta e possivelmente húmus, vivem dentro de madeira, em termiteiros construídos por outros cupins, ou constroem ninhos arborícolas e epígeos cartonados, de cor preta.

A infestação dos postes da rede elétrica urbana ocorre principalmente devido à falta de limpeza da vegetação em seu entorno. De modo geral, os postes estão localizados próximos e ao longo das calçadas, numa pequena faixa em frente às áreas residenciais ou comerciais sem nenhum cuidado do poder público ou do proprietário do lote. Assim, é comum sua base ser envolvida pela vegetação ou em contato com árvores utilizadas na arborização urbana (Figura 2), aumentando a possibilidade de infestação tanto pelo solo, via contato com a base, como pelos postes, cabos (telefônicos e elétricos) e cruzetas em contato com as árvores.

Os principais cupins pragas dos postes da rede elétrica da região do Alto Solimões pertencem à subfamília Nasutitermitinae. As espécies mais importantes são $N$. acangussu, $N$. nigriceps e $N$. ephratae. As espécies $C$. testaceus, $H$. tenuis e $T$. hispaniolae, também constituem pragas nesta região, mas de menor importância que as espécies do gênero Nasutitermes. Para reduzir os prejuízos é necessário que se faça a substituição dos postes de madeira por postes de concreto ou fibra, que mantenha suas bases sempre no limpo, além de não permitir que a parte superior dos postes e a fiação, ao longo de toda sua extensão, mantenha contato com árvores ou objetos que favoreçam a infestação por cupins.

\section{AGRADECIMENTOS}

Nós agradecemos ao Conselho Nacional de Desenvolvimento Científico e Tecnológico (CNPq) pelo apoio através do projeto de pesquisa MCT/CNPq/CT-Amazônia $\mathrm{N}^{\circ}$ 55/2008 e pela Bolsa de Iniciação Científica, concedida ao acadêmico do curso de Ciências Agrárias e do Ambiente Paulo V.C. de Oliveira. Agradecemos à profa. Shirlane Pantoja da Silva que nos auxílio em coletas e ao Sr. Nixon Franco Rabelo, identificador botânico do Instituto Natureza e Cultura - INC/UFAM.

\section{REFERÊNCIAS}

Abreu, R.L.S. \& M.A. Jesus, 2004. Durabilidade natural do estipe de pupunha (Bactris gasipaes Kunt, Arecaceae) II: Insetos. Acta Amazonica, 34: 459-465.

Acioli, A.N.S. \& E.P. Oliveira, 2010. Colonização por cupins (Insecta, Isoptera) em áreas reflorestadas da Mineração rio do Norte (MRN) - Porto Trombetas, Pará, Brasil, p. 39-51. In: E.G.C. Bermúdez, B. Ronchi-Teles \& R. Ale-Rocha (Org). Entomologia na Amazônia Brasileira. Manaus: INPA, 362p.

Ackerman, I.L., R. Constantino, H.G. Gauch-Jr,, J. Lehmann, S.J. Riha, \& E.C.M. Fernandes, 2009. Termite (Insecta: Isoptera) Species Composition in a Primary Rain Forest and Agroforests in Central Amazonia. Biotropica, 41: 226-233.

Bandeira, A.G., 1981. Ocorrência de cupins (Insecta, Isoptera) como pragas de mandioca em Bujarú, Pará. Acta Amazonica,

11: $149-152$.

Bandeira, A.G., 1998. Danos causados por cupins na Amazônia Brasileira, 87-98. In: Fontes, L.R. \& E. Berti-Filho (Eds.). Cupins: O Desafio do Conhecimento. Piracicaba: Fealq, 512p.

Bandeira, A.G., C.S. Miranda \& A. Vasconcellos, 1998. Danos causados por cupins em João Pessoa, Paraíba - Brasil, p. 7585. In: Fontes, L.R. \& E. Berti-Filho (Eds.). Cupins: O Desafio do Conhecimento. Piracicaba: Fealq, 512p.

Bandeira, A.G., J.I. Gomes, P.L.B. Lisboa, \& P.C.S. Souza, 1989. Insetos pragas de madeiras de edificações em Belém - Pará. EMBRAPA - CPATU (Boletim de Pesquisa No. 101), 25p.

Bandeira, A.G. \& L.R. Fontes, 1979. Nasutitermes acangussu, a new species of termite from Brazil (Isoptera, Termitidae, Nasutitermitinae). Revista Brasileira de Entomologia, 23: 119-122.

Beiguelman, B., 2002. Curso Prático de Bioestatística. $5^{\mathrm{a}}$ edição revisada. Fundação de Pesquisas Científicas de Ribeirão Preto. Ribeirão Preto, SP. 272p.

Coelho, M.R., E.C.C. Fidalgo, F.O. Araújo, H.G. Santos, M.L.M. Santos, D.V. Pérez \& F.M.S. Moreira, 2005. Levantamento pedológico de uma área-piloto relacionada ao projeto BiosBrasil (Conservation and sustainable management of below-ground biodiversity: Phase I), município de Benjamin Constant (AM): Janela 6. Rio de Janeiro: Embrapa (Boletim de Pesquisa e Desenvolvimento, 68), 95p.

Constantino, R., 2002. The pest termites of South America: taxonomy, distribution and status. Journal of Applied Entomology, 126: 355-365.

Constantino, R., 2001. Key to the soldiers of South American Heterotermes with a new species from Brazil (Isoptera: Rhinotermitidae). Insect Systematics and Evolution, 31: 463472.

Constantino, R., 1999. Chave ilustrada para identificação dos gêneros de cupins (Insecta: Isoptera) que ocorrem no Brasil. Arquivos de Zoologia, Museu de Zoologia da Universidade de São Paulo, 40: 387-448.

Constantino, R., 1998. Catalog of the living termites of the New World (Insecta: Isoptera). Arquivos de Zoologia, Museu de Zoologia da Universidade de São Paulo 35: 135-230.

Costa-Leonardo, A.M., 2002. Cupins-praga: Morfologia, biologia e controle. Rio Claro, SP. 128p.

Eleotério, E.S.R. \& B. Berti-Filho, 200o. Levantamento e identificação de cupins (Insecta: Isoptera) em área urbana de Piracicaba - SP. Ciência Florestal, 10: 125-139.

Emerson, A.E., 1925. The termites from Kartabo, Bartica District, Guyana. Zoologica 6: 291-459.

Fontes, L.R. \& R.L. Araujo, 1999. Os cupins, p. 35-90. In: Mariconi, F.A.M. (Coord.) Insetos e outros invasores de residências. Piracicaba: Fealg, 46op.

IBGE, 2011. Mapas integrados dos zoneamentos ecológicoeconômicos dos Estados da Amazônia Legal. Disponível em: $<$ www.ibge.gov.br/home/geociencias/geografia/mapas doc5.shtm>. Acesso: 20/03/2011.

Mathews, A.G.A., 1977. Studies on Termites from the Mato Grosso State, Brazil. Academia Brasileira de Ciências, Rio de Janeiro, 267p.

Milano, S. \& L.R. Fontes, 2002a. Cupim e Cidade: Implicações Ecológicas e Controle. São Paulo. 142p.

Milano, S. \& L.R. Fontes, 2002b. Termite pests and their control in urban Brazil. Sociobiology, 39: 163-177.

Nickle, D.A. \& M.S. Collins, 1992. The Termites of Panama (Isoptera), p. 208-241. In: Quintero, D. A. \& Aiello, A. (Eds.). Insects of Panama and Mesoamerica. Oxford University Press, New York, $1^{\text {st }}$ edition, 692p.

Nogueira, S.B. \& A.J. Souza, 1987. “Cupim do cerne”, Coptotermes testaceus (Isoptera: Rhinotermitidae), uma praga séria para eucaliptos nos cerrados. Brasil Florestal, 61: 27-29.

Robinson, W.H., 1996. Urban entomology: insect and mite pests in the human environment. London: Chapman \& Hall, 72p.

Thorne, B.L., 1980. Differences in nest architecture between the 
Neotropical arboreal termites Nasutitermes corniger and Nasutitermes ephratae (Isoptera: Termitidae). Psyche, 235243.

Thorne, B.L., M.I. Haverty \& M.S. Collins, 1994. Taxonomy and biogeography of Nasutitermes acajutlae and $N$. nigriceps (Isoptera: Termitidae) in the Caribbean and Cental America. Annals of the Entomological Society of America, 87: 762770 .
Waller, D.A. \& J.P. La Fage, 1986. Nutritional ecology of termites, p. 487-532. In: Slansky-Jr., F. \& J.C. Rodriguez (Eds.). Nutritional ecology of insects, mites, spiders and related invertebrates. New York: Wiley-Interscience Publication, $1016 \mathrm{p}$.

\section{Recebido em: 15/o3/2013}

Aceito em: 12/o6/2013

\section{Como citar este artigo:}

Acioli, A.N.S. \& P.V.C. Oliveira, 2013. Cupins (Isoptera) Invasores da Rede Elétrica em Áreas Urbanas na Região do Alto Solimões, Amazônia Ocidental, Brasil. EntomoBrasilis, 6(2): 150-156.

Acessível em: http://www.periodico.ebras.bio.br/ojs/index.php/ebras/article/view/332. doi:10.12741/ebrasilis.v6i2.332
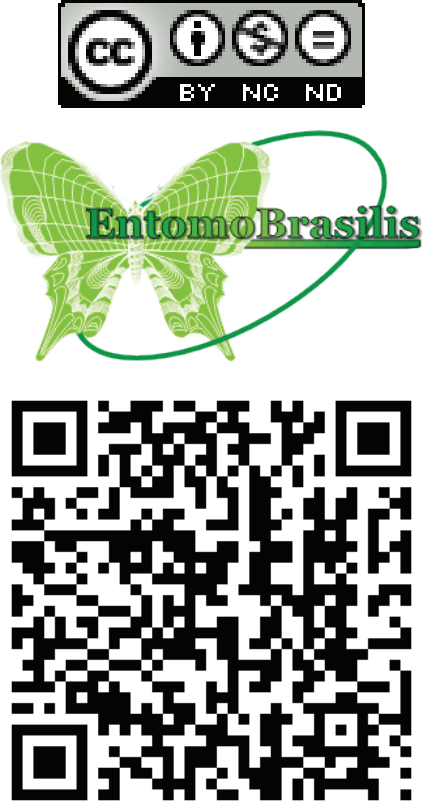